\title{
Entwurf von Fehlerisolationsbeobachtern für lineare Systeme mit unsicheren Parametern
}

\author{
Design of fault isolation observers for linear systems with uncertain parameters
}

Zusammenfassung: In diesem Beitrag wird ein Verfahren zum Entwurf von Fehlerisolationsbeobachtern für lineare Systeme mit unsicheren Parametern vorgestellt. Grundlage hierfür ist die Vollständige Modale Synthese, welche für nicht-quadratische Systeme mit mehr linear unabhängigen Messgrößen als potentiellen Fehlern eine Charakterisierung der sich ergebenden Freiheitsgrade liefert. Diese werden anschließend genutzt, um die strukturellen Anforderungen an die Fehlerisolation im Sinne unterschiedlicher Optimierungskriterien trotz vorhandener Unsicherheiten bestmöglich zu erfüllen und so die Robustheit bezüglich unsicherer Parameter zu erhöhen.

Schlüsselwörter: Beobachterbasierte Fehlerisolation, robuste Fehlerisolation, vollständige modale Synthese, unsicherheitsbehaftete Systeme.

\begin{abstract}
This paper presents a design for fault isolation observers for linear uncertain systems. It is based on a parametric eigenstructure assignment technique, which gives a comprehensive interpretation of the arising degrees of freedom in non-square systems with more linearly independent measurements than potential faults. These degrees of freedom are employed to fulfill the structural constraints of fault isolation as well as possible with respect to different optimization criteria. Therewith, robustness with respect to uncertain parameters is increased.
\end{abstract}

Keywords: Observer-based fault isolation, robust fault isolation, parametric eigenstructure assignment, uncertain systems.

*Korrespondenzautor: Arne Wahrburg, ABB AG, Ladenburg, E-Mail: arne.wahrburg@de.abb.com Jürgen Adamy: Technische Universität Darmstadt

\section{Einleitung}

Die modellbasierte Fehlerdiagnose ist ein in den vergangenen Jahren und Jahrzehnten intensiv erforschtes Gebiet der Regelungstechnik [3, 5, 12]. Dieses stete Interesse ist einerseits getrieben durch steigende Anforderungen an die Zuverlässigkeit immer komplexerer und diffizilerer Systeme. Andererseits haben leistungsfähigere Rechner die Entwicklung neuer Verfahren begünstigt. Wesentliche Forschungsrichtungen stellen Diagnosemethoden mit Paritätsgleichungen sowie identifikationsbasierte Verfahren dar [12]. Daneben haben sich beobachterbasierte Ansätze etabliert $[3,5]$, die auch in diesem Beitrag herangezogen werden.

Zwei wesentliche Aufgaben der Fehlerdiagnose sind die Fehlerdetektion und die Fehlerisolation. Während die Detektion darauf abzielt zu erkennen, ob ein oder mehrere Fehler auf das System einwirken, kann die Isolation als ein weiterführender Schritt verstanden werden. Ziel ist es hier, die auftretenden Fehler nicht nur zu erkennen, sondern auch zu lokalisieren.

Ein klassischer Ansatz zur Erfüllung dieser Aufgabe greift auf sogenannte Beobachterbänke zurück [3, 8]. Dabei werden verschiedene Beobachter parallel implementiert und die Isolation erfolgt anhand einer Auswertungslogik. In diesem Beitrag wird ein anderer Ansatz verfolgt, welcher für eine eingeschränkte Klasse von linearen Systemen erstmals in [16] vorgeschlagen wurde. Ziel ist es, einen einzigen Beobachter derart auszulegen, dass die generierten Residuen neben einer Detektion der Fehler auch eine Isolation ermöglichen. In jüngerer Vergangenheit wurden verschiedene Verfahren zum Entwurf solcher Fehlerisolationsbeobachter (FIOs) vorgeschlagen, welche z. T. die Klasse der handhabbaren linearen Systeme erweitern. Während [2] auf der Vorgabe einer geeigneten Eigenstruktur des Beobachters beruht, wird in [5, Abschnitt 13.3.3] eine 
geometrische Lösung angegeben. Die Verfahren in $[13,15]$ basieren auf Frequenzbereichsbetrachtungen. In [30] wird die Dualität des Problems zum Entwurf linearer Entkopplungsregler [6, 27] ausgenutzt, um FIOs mittels Vollständiger Modaler Synthese [26] zu entwerfen.

Ein Kernproblem aller modellbasierter Ansätze ist die Robustheit der Fehlerisolation bezüglich exogener Störeinflüsse sowie Ungenauigkeiten in der Modellierung. Die Robustheit der Residuengenerierung bezüglich äußerer Störungen wird für FIOs u.a. in $[2,15,30]$ betrachtet. Hinsichtlich unsicherer Systemparameter finden sich jedoch weniger Resultate in der Literatur. Ein gängiger Ansatz beruht darauf, die parametrischen Unsicherheiten in exogene Störungen umzuformulieren [24] und auf dieser Basis das System für den Entwurf als exakt bekannt vorauszusetzen. Dies kann jedoch wie in [5, Abschnitt 8.2] erörtert zu konservativen Ergebnissen führen. Einen weiteren Lösungsansatz stellt die Formulierung eines Model-Matching-Problems dar. Dabei wird versucht, das Übertragungsverhalten des Residuengenerators trotz vorhandener unsicherer Systemparameter möglichst gut an ein gegebenes Referenzmodell anzunähern. Dieser Ansatz wird z. B. in [21, 33] verfolgt. Er erfordert jedoch zusätzlichen Aufwand, da ein geeignetes Referenzmodell gefunden werden muss [9].

In diesem Artikel wird ein neues, auf der Vollständigen Modalen Synthese basierendes Verfahren vorgeschlagen. Es greift die Grundidee des Beitrages [29] auf und erweitert die Ergebnisse. So ermöglicht der Ansatz die direkte Berücksichtigung unsicherer Systemparameter, eine Umformulierung als virtuelle exogene Störungen ist nicht notwendig. Weiterhin ist kein Referenzmodell nötig. Außerdem ist durch die Methode gewährleistet, dass für das nominale System, d.h. ohne Unsicherheiten in den Systemparametern, eine exakte Fehlerisolation gelingt. Die verbleibenden Freiheitsgrade werden zur Steigerung der Robustheit bezüglich unsicherer Parameter genutzt.

Der Beitrag ist wie folgt aufgebaut. In Abschnitt 2 wird neben der Notation die Problemstellung ausführlich erörtert. Anschließend wird in Abschnitt 3 der in [30] entwickelte Entwurf von FIOs für die nominale Systemdynamik zusammengefasst. Dabei bietet die Vollständige Modale Synthese im Gegensatz zu anderen Methoden eine anschauliche Interpretation der verbleibenden Freiheitsgrade. Diese werden in Abschnitt 4 verwendet, um die Anforderungen an die Eigenstruktur des Gesamtsystems möglichst gut im Sinne der Fehlerisolation zu erfüllen. Das Verfahren wird exemplarisch für ein akademisches Beispielsystem angewendet (Abschnitt 5), bevor der Beitrag in Abschnitt 6 zusammengefasst wird.

\section{Grundlagen}

\subsection{Notation}

Für eine positiv (negativ) definite, symmetrische Matrix $\boldsymbol{A}=\boldsymbol{A}^{\top} \in \mathbb{R}^{n \times n}$ wird $\boldsymbol{A} \succ(\prec) \mathbf{0}$ geschrieben. Elemente der Matrix, welche sich unmittelbar aus der Symmetrie ergeben, werden mit $\star$ abgekürzt. Für eine Diagonalmatrix $S \in \mathbb{R}^{n \times n}$ mit den Diagonalelementen $s_{i}$ wird $S=$ $\operatorname{diag}\left(s_{1}, \ldots, s_{n}\right)$ geschrieben. Die Vektoren $\boldsymbol{p}_{i}\left(\boldsymbol{p}_{j}^{\top}\right)$ bezeichnen die $i$-te Spalte ( $j$-te Zeile) einer Matrix $\boldsymbol{P} \in$ $\mathbb{R}^{n \times m}$. Ist $n>m$ und $r=\operatorname{rang}(\boldsymbol{P})$, so existiert nach dem Rangsatz [22] ein linkes orthogonales Komplement ${ }^{\perp} \boldsymbol{P} \in$ $\mathbb{R}^{(m-r) \times n}$, welches ${ }^{\perp} \boldsymbol{P} \cdot \boldsymbol{P}=\mathbf{0}$ erfüllt. Weiterhin ist $\boldsymbol{P}$ linksinvertierbar und es gilt $\boldsymbol{P}^{+} \cdot \boldsymbol{P}=\boldsymbol{I}_{m}$. Darüber hinaus wird in diesem Beitrag

$$
\phi_{i}=\left[\begin{array}{lllllll}
0 & \cdots & 0 & 1 & 0 & \cdots & 0
\end{array}\right]^{\top}
$$

für einen Einheitsvektor geschrieben, der an der $i$-ten Stelle das Element 1 enthält.

\subsection{Problemstellung}

Betrachtet werden lineare, zeit-invariante Systeme der Form

$$
\begin{aligned}
& \dot{x}=A_{\xi} x+B_{\xi} u+E_{a, \xi} f, \\
& y=C_{\xi} x+D_{\xi} u+E_{s, \xi} f
\end{aligned}
$$

mit dem Zustandsvektor $\boldsymbol{x} \in \mathbb{R}^{n}$, Eingangsgrößen $\boldsymbol{u} \in \mathbb{R}^{n_{u}}$, messbaren Ausgangsgrößen $y \in \mathbb{R}^{n_{y}}$, dem Fehlervektor $f \in \mathbb{R}^{n_{f}}$ und Matrizen passender Dimensionen. In $f$ sind sowohl Fehler zusammengefasst, die über $\boldsymbol{E}_{a, \xi}$ auf die Dynamik der Strecke wirken (z. B. Aktorfehler), als auch solche, welche aufgrund eines Durchgriffs über $\boldsymbol{E}_{s, \xi}$ direkt auf die Messgleichung Einfluss nehmen (Sensorfehler). Es ist also eine direkte Betrachtung von Sensorfehlern möglich, eine Umformulierung als Pseudoaktorfehler verbunden mit dynamischen Systemerweiterungen wie in [23] ist nicht notwendig. Betrachtet werden nicht-quadratische Systeme mit $n_{y}>n_{f}$.

Es wird angenommen, dass die Systemmatrizen nicht exakt bekannt, sondern mit Unsicherheiten behaftet sind. Dies kann zum Beispiel durch nicht genau bekannte Systemparameter hervorgerufen werden. Um diesen Sachverhalt mathematisch zu beschreiben, wird angenommen, dass für die Systemmatrizen in (2)

$$
\left[\begin{array}{ccc}
\boldsymbol{A}_{\boldsymbol{\xi}} & \boldsymbol{B}_{\boldsymbol{\xi}} & \boldsymbol{E}_{a, \xi} \\
\boldsymbol{C}_{\xi} & \boldsymbol{D}_{\xi} & \boldsymbol{E}_{s, \xi}
\end{array}\right] \in \mathcal{M},
$$




$$
\mathcal{M}=\left\{\left[\begin{array}{ccc}
\boldsymbol{A}_{\boldsymbol{\xi}} & \boldsymbol{B}_{\boldsymbol{\xi}} & \boldsymbol{E}_{a, \xi} \\
\boldsymbol{C}_{\boldsymbol{\xi}} & \boldsymbol{D}_{\boldsymbol{\xi}} & \boldsymbol{E}_{s, \xi}
\end{array}\right]=\sum_{l=1}^{N} \xi_{l}^{\left[\begin{array}{ccc}
\boldsymbol{A}_{l} & \boldsymbol{B}_{l} & \boldsymbol{E}_{a, l} \\
\boldsymbol{C}_{l} & \boldsymbol{D}_{l} & \boldsymbol{E}_{s, l}
\end{array}\right]} \boldsymbol{M}_{l}\right.
$$

$$
\xi_{l} \geq 0, \sum_{l=1}^{N} \xi_{l}=1
$$

gilt. Die Systemmatrizen lassen sich also jeweils als konvexe Kombination von $N$ Eckpunkten eines Polytops darstellen. Darüber hinaus existiert ein nominales Modell

$$
\begin{aligned}
& \dot{x}=A_{0} x+B_{0} u+E_{a, 0} f, \\
& y=C_{0} x+D_{0} u+E_{s, 0} f,
\end{aligned}
$$

für welches trivialerweise $\boldsymbol{M}_{0} \in \mathcal{M}$ gelten muss. Abbildung 1 verdeutlicht die Annahmen bezüglich der Systembeschreibung beispielhaft für ein System mit $N=4$ extremalen Systemen.

Zur Detektion und Isolation der Fehler $\boldsymbol{f}$ wird ein Beobachter der Form

$$
\begin{aligned}
\dot{\hat{x}} & =A_{0} \hat{x}+B_{0} u+L\left(y-C_{0} \hat{x}-D_{0} u\right), \\
r & =V\left(y-C \hat{x}-D_{0} u\right)
\end{aligned}
$$

angesetzt, welcher die Residuen $\boldsymbol{r} \in \mathbb{R}^{n_{f}}$ erzeugt. Fasst man Strecken- und Beobachterzustandsvektor zusammen zu $\tilde{\boldsymbol{x}}=\left[\begin{array}{ll}\boldsymbol{x}^{\top} & \hat{\boldsymbol{x}}^{\top}\end{array}\right]^{\top}$ und setzt (2) und (5) ein, so ergibt sich für jeden Eckpunkt des Polytops

$$
\begin{aligned}
& \dot{\tilde{x}}=\left[\begin{array}{c}
\dot{\boldsymbol{x}} \\
\dot{\hat{x}}
\end{array}\right]=(\underbrace{\left[\begin{array}{cc}
A_{l} & 0 \\
0 & A_{0}
\end{array}\right]}_{\tilde{A}_{l}}-\underbrace{\left[\begin{array}{c}
0 \\
-L
\end{array}\right]}_{\tilde{L}} \underbrace{\left[\begin{array}{ll}
C_{l} & -C_{0}
\end{array}\right]}_{\tilde{C}_{l}}) \tilde{\boldsymbol{x}} \\
& +(\underbrace{\left[\begin{array}{c}
\boldsymbol{B}_{l} \\
\boldsymbol{B}_{0}
\end{array}\right]}_{\tilde{\boldsymbol{B}}_{l}}-\underbrace{\left[\begin{array}{c}
\mathbf{0} \\
-\boldsymbol{L}
\end{array}\right]}_{\tilde{\boldsymbol{L}}} \underbrace{\left(\boldsymbol{D}_{l}-\boldsymbol{D}_{0}\right)}_{\tilde{\boldsymbol{D}}_{l}}) \boldsymbol{u}, \\
& +(\underbrace{\left[\begin{array}{c}
\boldsymbol{E}_{a, l} \\
\mathbf{0}
\end{array}\right]}_{\tilde{E}_{a, l}}-\underbrace{\left[\begin{array}{c}
\mathbf{0} \\
-\boldsymbol{L}
\end{array}\right]}_{\tilde{L}} \underbrace{\boldsymbol{E}_{s, l}}_{\tilde{E}_{s, l}}) \boldsymbol{f}, \\
& \boldsymbol{r}=\boldsymbol{V} \underbrace{\left[\begin{array}{ll}
C_{l}-C_{0}
\end{array}\right]}_{\tilde{C}_{l}} \tilde{\boldsymbol{x}}+\boldsymbol{V} \underbrace{\left(D_{l}-D_{0}\right)}_{\tilde{D}_{l}} \boldsymbol{u}+\boldsymbol{V} \underbrace{\boldsymbol{E}_{s, l}}_{\tilde{E}_{s, l}} \boldsymbol{f} \text {. }
\end{aligned}
$$

Hiermit lassen sich die Übertragungsmatrizen

$$
\begin{aligned}
\boldsymbol{G}_{r f, l}(s)= & \boldsymbol{V} \tilde{\boldsymbol{C}}_{l}\left(s \boldsymbol{I}_{2 n}-\left(\tilde{\boldsymbol{A}}_{l}-\tilde{\boldsymbol{L}}_{l}\right)\right)^{-1} \cdot \ldots \\
& \left(\tilde{\boldsymbol{E}}_{a, l}-\tilde{\boldsymbol{L}} \tilde{\boldsymbol{E}}_{s, l}\right)+\boldsymbol{V} \tilde{\boldsymbol{E}}_{s, l}, \\
\boldsymbol{G}_{\boldsymbol{r u}, l}(s)=\boldsymbol{V} & \tilde{\boldsymbol{C}}_{l}\left(s \boldsymbol{I}_{2 n}-\left(\tilde{\boldsymbol{A}}_{l}-\tilde{\boldsymbol{L}} \tilde{\boldsymbol{C}}_{l}\right)\right)^{-1} \cdot \ldots \\
& \left(\tilde{\boldsymbol{B}}_{l}-\tilde{\boldsymbol{L}} \tilde{\boldsymbol{D}}_{l}\right)+\boldsymbol{V} \tilde{\boldsymbol{D}}_{l}
\end{aligned}
$$

ableiten, welche die Wirkung der Fehler $\boldsymbol{f}$ bzw. der Stellgrößen $\boldsymbol{u}$ auf die Residuen beschreiben. Idealziel ist es, nicht nur für die Eckpunkte des Polytops sondern für al-

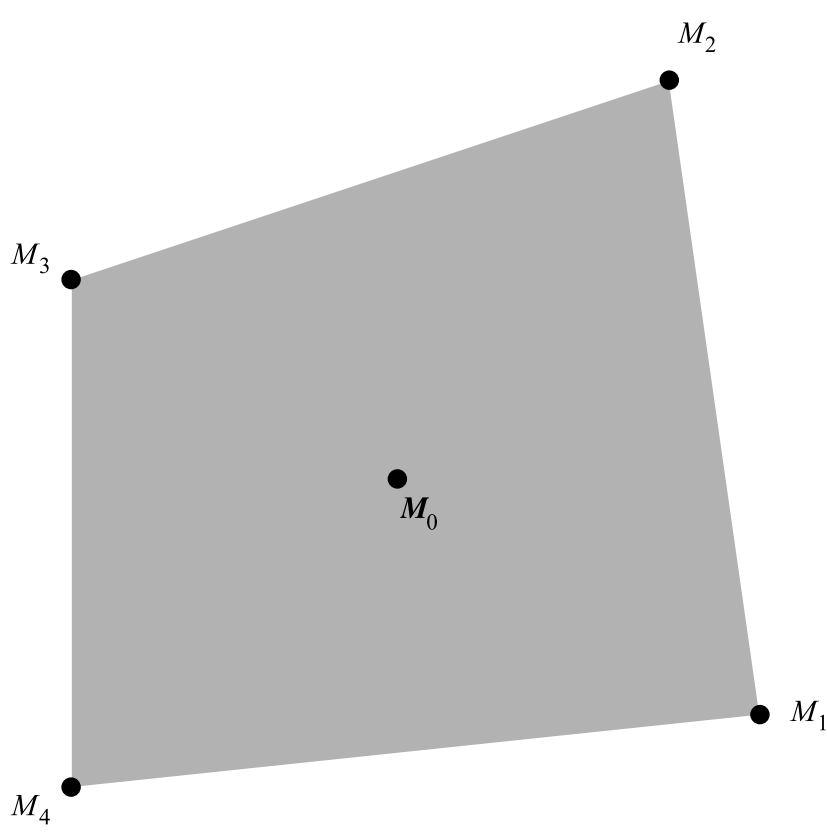

Abbildung 1: Beschreibung eines unsicherheitsbehafteten Systems als Polytop.

le Systeme in $\mathcal{M}$ durch eine geeignete Parametrierung von $\boldsymbol{L}$ und $\boldsymbol{V}$ Übertragungsverhalten der Form

$$
\begin{aligned}
& \boldsymbol{G}_{r f, \xi}(s)=\operatorname{diag}\left(g_{1,1}(s), \ldots, g_{n_{f}, n_{f}}(s)\right), \\
& \boldsymbol{G}_{r u, \xi}(s)=\mathbf{0} \forall s \in \mathbb{C}
\end{aligned}
$$

zu erzielen. In diesem Fall wirken sich die Stellgrößen $\boldsymbol{u}$ nicht auf die Residuen aus und die Fehler $f$ nehmen dergestalt Einfluss, dass ein einzelnes Residuum $r_{i}$ ausschließlich von einem ihm zugeordneten Fehler $f_{i}$ angeregt wird. Damit lässt sich der entsprechende Fehler nicht nur detektieren, sondern auch von den übrigen Fehlern isolieren, selbst wenn diese zeitgleich aktiv sein sollten.

Es ist offensichtlich, dass (8) im Allgemeinen nicht mit einem Beobachter der Form (5) für alle Systeme in $\mathcal{M}$ erreicht werden kann. Im Rahmen dieses Beitrages wird daher folgender Ansatz verfolgt: Die Beobachterparametrierung $(\boldsymbol{L}, \boldsymbol{V})$ soll für das durch $\boldsymbol{M}_{0}$ gegebene nominale System eine exakte Fehlerisolation gewährleisten, d.h. $\boldsymbol{G}_{r f, 0}(s)$ und $\boldsymbol{G}_{r u, 0}(s)$ sollen (8) erfüllen. Für alle übrigen Systeme in $\mathcal{M}$ werden dann die verbleibenden Freiheitsgrade genutzt, um (8) möglichst gut zu erfüllen. Auf die dabei zugrunde gelegten Optimierungskriterien wird in Abschnitt 4 näher eingegangen.

Anmerkung 1. In der Praxis unterliegen Systeme wie bereits erörtert nicht nur unsicheren Parametern, sondern auch exogenen Störungen wie beispielsweise Sensorrauschen. Diese werden im Systemmodell (2) nicht berücksichtigt, da der Fokus dieses Beitrages auf den bisher 
im Bereich der beobachterbasierten Fehlerisolation weniger intensiv untersuchten parametrischen Unsicherheiten liegt. Der Einfluss exogener Störungen auf die Residuengenerierung lässt sich jedoch ohne Weiteres in die vorgestellte Entwurfsmethodik einbeziehen. Hierauf wird in Abschnitt 4 in Anmerkung 3 näher eingegangen.

Für den Entwurf der Fehlerisolationsbeobachter werden die Fehlerdetektionsindizes $\delta_{i}, i=1, \ldots, n_{f}$ und die Fehlerdetektionsmatrix $\boldsymbol{D}^{*}$ eingeführt. In Erweiterung der Definition aus [16] sind die Fehlerdetektionsindizes gegeben durch

$$
\delta_{i}= \begin{cases}0 & , \boldsymbol{e}_{s_{i}, 0} \neq \mathbf{0}, \\ \min _{k}\left\{k \geq 1: \boldsymbol{C}_{0} \boldsymbol{A}_{0}^{k-1} \boldsymbol{e}_{a_{i}, 0} \neq \mathbf{0}\right\} & , \boldsymbol{e}_{s_{i}, 0}=\mathbf{0},\end{cases}
$$

wobei $\boldsymbol{e}_{a_{i}, 0}$ und $\boldsymbol{e}_{s_{i}, 0}$ die $i$-te Spalte der nominalen Aktorbzw. Sensorfehlermatrix beschreiben. Anschaulich gesprochen gibt $\delta_{i}$ an, wie oft der Ausgangsvektor $y$ zeitlich abgeleitet werden muss, damit der $i$-te Fehler explizit im sich ergebenden Ausdruck auftaucht. Summation über alle Fehlerindizes liefert den Gesamtfehlerindex

$$
\delta=\sum_{i=1}^{n_{f}} \delta_{i} .
$$

Die Fehlerindizes bestimmen darüber hinaus die Fehlerdetektionsmatrix mit, welche definiert ist als

$$
\begin{aligned}
\boldsymbol{D}^{*} & =\left[\begin{array}{lll}
\boldsymbol{d}_{1}^{*} & \cdots & \boldsymbol{d}_{n_{f}}^{*}
\end{array}\right], \\
\boldsymbol{d}_{i}^{*} & = \begin{cases}\boldsymbol{e}_{s_{i}, 0} & , \delta_{i}=0, \\
\boldsymbol{C}_{0} A_{0}^{\delta_{i}-1} \boldsymbol{e}_{a_{i}, 0} & , \delta_{i} \geq 1 .\end{cases}
\end{aligned}
$$

Mithilfe dieser Definitionen lassen sich die Annahmen formulieren, welche im vorliegenden Beitrag hinsichtlich des Systems getroffen werden.

Annahme 1. Das Paar $\left(A_{0}, C_{0}\right)$ ist vollständig beobachtbar.

Annahme 2. Die Matrix $\boldsymbol{A}_{\xi}$ ist für alle $\boldsymbol{\xi}$ eine HurwitzMatrix.

Annahme 3. Die Ausgangsmatrix $C_{0}$ hat vollen Rang.

Annahme 4. Die Fehlerdetektionsmatrix $D^{*}$ hat vollen Rang.

Annahme 5. Das System (2) weist genau $\mu$ invariante Nullstellen auf, die alle in der offenen linken s-Halbebene liegen.

Während Annahme 1 sicherstellt, dass alle Beobachtereigenwerte beliebig platziert werden können, garantiert Annahme 2 die Stabilität des Systems für alle möglichen Wer- te der unsicheren Parameter. Durch Annahme 3 werden linear abhängige und damit redundante Messungen ausgeschlossen. Die Annahmen 4 und 5 stellen für quadratische Systeme mit $n_{y}=n_{f}$ wie in [19] für das duale Problem des Entkopplungsreglerentwurfs gezeigt notwendige und hinreichende Bedingungen für die Existenz eines stabilen FIOs dar. Lediglich für den Spezialfall von Systemen mit nicht-verkoppelnden Nullstellen [14, 18] ist Annahme 5 nicht notwendig. Dieser Fall wird hier jedoch nicht näher betrachtet. Für nicht-quadratische Systeme mit $n_{y}>n_{f}$ sind die Bedingungen aus Annahme 4 und 5 hinreichend für den Entwurf stabiler FIOs. Ebenso wie für das Problem des Entkopplungsreglerentwurfs [4, 11, 31, 32] existieren für den FIO-Entwurf in nicht-quadratischen Systemen bisher keine allgemeingültigen notwendigen und heinreichenden Bedingungen.

$\mathrm{Zu}$ bemerken ist weiterhin, dass sich die Annahmen 1, 3, 4 und 5 ausschließlich auf das nominale System (4) beziehen. Für die Eckpunkte des in (3) definierten Polytops müssen sie prinzipiell nicht notwendigerweise erfüllt sein, jedoch sind dann keine guten Ergebnisse hinsichtlich der Robustheit zu erwarten.

\section{Entwurf eines FIO für das nominale System}

In diesem Abschnitt wird der Entwurf eines FIOs für das nominale System kurz zusammengefasst. Das Entwurfsverfahren basiert auf der Vollständigen Modalen Synthese und nutzt die Dualität von FIOs für eine nominale Systemdynamik und Entkopplungsregelungen [27] aus. Details zum vorgestellten Entwurf finden sich in [30].

Betrachtet man die Dynamik des Gesamtsystems aus Strecke und Beobachter für den nominalen Fall, so lässt sich durch Einführen des Beobachterfehlers $\boldsymbol{\zeta}=\boldsymbol{x}-\hat{\boldsymbol{x}}$ ein virtuelles System

$$
\begin{aligned}
& \dot{\zeta}=\left(A_{0}-L C_{0}\right) \zeta+\left(E_{a, 0}-L E_{s, 0}\right) f \\
& r=V C_{0} \zeta+V E_{s, 0} f
\end{aligned}
$$

herleiten. Für den Entwurf wird nun die folgende vereinfachende Annahme getroffen.

Annahme 6. Die vorgegebenen Beobachtereigenwerte $\lambda_{B_{1}}, \ldots, \lambda_{B_{n}}$ sind paarweise verschieden.

Diese Annahme stellt wie in $[17,26]$ gezeigt keine Einschränkung der Allgemeinheit dar, da sie bei einer entsprechenden Erweiterung des Verfahrens fallen gelassen werden kann. 
Unter der Annahme 6 lässt sich die aus (12) resultierende Übertragungsmatrix in Partialbruchdarstellung schreiben als

$$
\boldsymbol{G}_{r f, 0}(s)=\sum_{k=1}^{n} \frac{\boldsymbol{V} \boldsymbol{C}_{0} \boldsymbol{v}_{B_{k}} \cdot \boldsymbol{w}_{B_{k}}^{\top}\left(\boldsymbol{E}_{a, 0}-\boldsymbol{L} \boldsymbol{E}_{s, 0}\right)}{s-\lambda_{B_{k}}}+\boldsymbol{V} \boldsymbol{E}_{s, 0} .
$$

Dabei sind $\boldsymbol{v}_{B_{k}}=\boldsymbol{v}_{B_{k}, 0}$ und $\boldsymbol{w}_{B_{k}}^{\top}=\boldsymbol{w}_{B_{k}, 0}^{\top}$ die Rechts- bzw. Linkseigenvektoren von $\boldsymbol{A}_{0}-\boldsymbol{L C}_{0}$ zum Beobachtereigenwert $\lambda_{B_{k}}$. Grundidee des in [27] vorgestellten und in [30] aufgegriffenen Ansatzes ist es, die Eigenstruktur des Systems (12) durch die Wahl von $\boldsymbol{L}$ und $\boldsymbol{V}$ geeignet zu beeinflussen.

Für die ersten $\delta$ Beobachtereigenwerte wird eine Doppelindizierung $\lambda_{B_{i j}}$ eingeführt. Dabei beschreibt $i=$ $1, \ldots, n_{f}$ den Übertragungskanal, dem der Eigenwert $\lambda_{B_{i j}}$ zugeordnet wird. Der Index $j=1, \ldots, \delta_{i}$ nummeriert die Pole im $i$-ten Übertragungskanal.

Wegen des dyadischen Produktes in (13) taucht der Eigenwert $\lambda_{B_{i j}}$ ausschließlich in der $i$-ten Spalte von $\boldsymbol{G}_{\boldsymbol{r} f, 0}(s)$ auf, wenn $\boldsymbol{w}_{B_{i j}}^{\top}\left(\boldsymbol{E}_{a, 0}-\boldsymbol{L E}_{s, 0}\right)=\boldsymbol{\phi}_{i}^{\top}$ gilt. Dabei ist $\phi_{i}^{\top}$ durch (1) gegeben. Die verbleibenden Beobachtereigenwerte $\lambda_{B_{k}}, k=\delta+1, \ldots, n$ sind nach dem GilbertKriterium [10] bezüglich des Paares $\left(\boldsymbol{A}_{0}-\boldsymbol{L} \boldsymbol{C}_{0}, \boldsymbol{E}_{a, 0}-\boldsymbol{L} \boldsymbol{E}_{s, 0}\right)$ nicht-steuerbar, falls $\boldsymbol{w}_{B_{k}}^{\top}\left(\boldsymbol{E}_{a, 0}-\boldsymbol{L} \boldsymbol{E}_{s, 0}\right)=\mathbf{0}^{\top}$ gilt. Definitionsgemäß gilt für die Linkseigenvektoren $\boldsymbol{w}_{B_{i j}}^{\top}\left(A_{0}-L C_{0}\right)=$ $\lambda_{B_{i j}} \boldsymbol{w}_{B_{i j}}^{\top}$. Unter Zuhilfenahme der Parametervektoren

$$
\boldsymbol{p}_{i j}^{\top}=-\boldsymbol{w}_{B_{i j}}^{\top} \boldsymbol{L}, \text { bzw. } \boldsymbol{p}_{k}^{\top}=-\boldsymbol{w}_{B_{k}}^{\top} \boldsymbol{L},
$$

die bereits in [25] für den modalen Beobachterentwurf vorgeschlagen wurden, lässt sich damit die Beziehung

$$
\boldsymbol{w}_{B_{i j}}^{\top}\left(\lambda_{B_{i j}} \boldsymbol{I}_{n}-\boldsymbol{A}_{0}\right)=-\boldsymbol{w}_{B_{i j}}^{\top} \boldsymbol{L} \boldsymbol{C}_{0}=\boldsymbol{p}_{i j}^{\top} \boldsymbol{C}_{0}
$$

ableiten. Daraus ergibt sich wie in [30] beschrieben ein konstruktives Verfahren zur Berechnung der Beobachtermatrix $\boldsymbol{L}$ herleiten.

Die Nachfiltermatrix $\boldsymbol{V}$ wird anschließend so ausgelegt, dass sich im stationären Fall, d.h. im LaplaceBereich für $s=0$, eine Diagonalmatrix $\boldsymbol{G}_{r f, 0}(s=0)$ ergibt. Wie für das duale Problem des Entkopplungsreglerentwurfs [18] lässt sich zeigen, dass dies unter den getroffenen Annahmen immer möglich ist und dass die gewonnene Nachfiltermatrix $\boldsymbol{V}$ eine vollständige Diagonalisierung von $G_{r f, 0}(s)$ für alle $s \in \mathbb{C}$ gewährleistet.

Der folgende Satz fasst das Entwurfsverfahren für die nominale Systemdynamik in Systemen mit $n_{y} \geq n_{f}$ zusammen.

Satz 1. Gegeben sei das nominale System (4), welches die Annahmen 1-6 erfüllt. Weiterhin seien $\boldsymbol{z}_{i j}^{\top} \in \mathbb{R}^{n_{y}-n_{f}}, \quad \boldsymbol{z}_{i n, m}^{\top} \neq \mathbf{0}^{\top} \in \mathbb{R}^{n_{y}-n_{f}+1}, \quad \boldsymbol{z}_{k}^{\top} \neq \mathbf{0}^{\top} \in \mathbb{R}^{n_{y}-n_{f}}$ beliebige Zeilenvektoren, $Z_{V} \in \mathbb{R}^{n_{f} \times\left(n_{y}-n_{f}\right)}$ eine beliebige Matrix und $S=\operatorname{diag}\left(z_{1}, \ldots, z_{n_{f}}\right)$ eine beliebige Diagonalmatrix. Werden die $\mu$ Beobachtereigenwerte $\lambda_{B_{\delta+1}}, \ldots, \lambda_{B_{\delta+\mu}}$ entsprechend den invarianten Nullstellen des Systems gewählt, dann resultieren alle durch

$$
\begin{aligned}
& \boldsymbol{L}=-\boldsymbol{W}_{B}^{-1} \boldsymbol{P}, \\
& {\left[\begin{array}{ll}
\boldsymbol{w}_{B_{i j}}^{\top} & \boldsymbol{p}_{i j}^{\top}
\end{array}\right]=\left[\begin{array}{ll}
\mathbf{0}^{\top} & \boldsymbol{\phi}_{i}^{\top}
\end{array}\right] \boldsymbol{\Pi}_{i j}^{+}+\boldsymbol{z}_{i j}^{\top} \cdot{ }^{\perp} \boldsymbol{\Pi}_{i j},} \\
& i=1, \ldots, n_{f}, j=1, \ldots, \delta_{i}, \\
& {\left[\begin{array}{ll}
\boldsymbol{w}_{B_{m}}^{\top} & \boldsymbol{p}_{m}^{\top}
\end{array}\right]=\boldsymbol{z}_{i n, m}^{\top} \cdot{ }^{\perp} \boldsymbol{\Pi}_{m}, m=\delta+1, \ldots, \delta+\mu,} \\
& {\left[\begin{array}{ll}
\boldsymbol{w}_{B_{k}}^{\top} & \boldsymbol{p}_{k}^{\top}
\end{array}\right]=\boldsymbol{z}_{k}^{\top} \cdot{ }^{\perp} \boldsymbol{\Pi}_{k}, k=\delta+\mu+1, \ldots, n,} \\
& \boldsymbol{V}=-\boldsymbol{S} \boldsymbol{\Theta}^{+}-\boldsymbol{Z}_{V} \cdot{ }^{\perp} \boldsymbol{\Theta}, \\
& \boldsymbol{\Theta}=\boldsymbol{C}_{0}\left(\boldsymbol{A}_{0}-\boldsymbol{L C}_{0}\right)^{-1}\left(\boldsymbol{E}_{a, 0}-\boldsymbol{L} \boldsymbol{E}_{s, 0}\right)-\boldsymbol{E}_{s, 0}
\end{aligned}
$$

definierten Beobachterparametrierungen $(\boldsymbol{L}, \boldsymbol{V})$ in einer diagonalisierten Übertragungsmatrix $\boldsymbol{G}_{r f, 0}(s)$ der Form

$$
\begin{aligned}
\boldsymbol{G}_{r f, 0}(s) & =\operatorname{diag}\left(g_{1,1}(s), \ldots, g_{n_{f}, n_{f}}(s)\right), \\
g_{i, i}(s) & =\frac{\tilde{z}_{i}}{\left(s-\lambda_{B_{i 1}}\right) \cdot \ldots \cdot\left(s-\lambda_{B_{i \delta_{i}}}\right)}, \\
\tilde{z}_{i} & =(-1)^{n} \cdot z_{i} \cdot \prod_{j=1}^{\delta_{i}} \lambda_{B_{i j}} .
\end{aligned}
$$

Weiterhin gilt für alle diese Parametrierungen $\boldsymbol{G}_{r u, 0}(s)=\mathbf{0}$.

Beweis. Der Beweis beruht wie in obigen Überlegungen angedeutet im Wesentlichen auf einer Partialbruchzerlegung von $G_{r f, 0}(s)$ und der parametrischen Eigenstrukturvorgabe der Matrix $\boldsymbol{A}_{0}-\boldsymbol{L} \boldsymbol{C}_{0}$. Für Details wird auf die ausführliche Herleitung in [30] verwiesen.

Mit Hilfe der Matrix $\boldsymbol{S}$ lässt sich die stationäre Verstärkung der einzelnen Fehler festlegen. Oftmals wird $z_{i}=1, i=$ $1, \ldots, n_{f}$ gewählt, da dann für den nominalen Fall nicht nur eine Fehlerisolation, sondern auch eine Fehleridentifikation erreicht wird. Da das Residuum im stationären Fall dem Fehler entspricht, lässt sich eine Aussage über die Größe des jeweiligen Fehlers treffen. Als verbleibende Freiheitsgrade zur Steigerung der Robustheit verbleiben somit $\boldsymbol{z}_{i j}^{\top}, \boldsymbol{z}_{\mathrm{in}, m}^{\top}, \lambda_{B_{k}}, \boldsymbol{z}_{k}^{\top}$ und $\boldsymbol{Z}_{V}$.

Betrachtet man die Dimensionen dieser Größen, so wird deutlich, dass im quadratischen Fall, d. h. für $n_{y}=n_{f}$ keinerlei Entwurfsfreiheitsgrade verbleiben. Es ist dann lediglich der zum Skalar entartete Zeilenvektor $\boldsymbol{z}_{\text {in,m }}^{\top}$ vor- 
handen. Da die Eigen- bzw. Parametervektoren jedoch skalierungsinvariant sind [26], ergibt sich daraus kein Freiheitsgrad. Satz 1 schließt den quadratischen Fall als Spezialfall mit ein. Dieser Spezialfall stellt das duale Ergebnis zum bekannten Entkopplungsreglerentwurf für Systeme mit ebensovielen Eingangs- wie Ausgangsgrößen dar [27]. Im nicht-quadratischen Fall $n_{y}>n_{f}$ gibt es jedoch gemäß Satz 1 unendlich viele Beobachterparametrierungen, welche die Entwurfsziele (8) für das nominale System erfüllen. Durch $\boldsymbol{z}_{i j}^{\top}, \boldsymbol{z}_{\text {in, } m}^{\top}$ und $\boldsymbol{z}_{k}^{\top}$ lassen sich die Linkseigenvektoren und Parametervektoren innerhalb des Linksnullraumes der Rosenbrock'schen Systemmatrix variieren. Außerdem sind diejenigen Eingangsentkopplungsnullstellen, welche keine invarianten Nullstellen sind, im nicht-quadratischen System beliebig wählbar. Die Beobachtereigenwerte $\lambda_{B_{k}}$ können somit beliebig platziert werden. Nach der Bestimmung der Beobachtermatrix $\boldsymbol{L}$ bieten sich schließlich durch $\boldsymbol{Z}_{V}$ zusätzliche Freiheitsgrade bei der Wahl der Nachfiltermatrix $\boldsymbol{V}$.

\section{Optimierung der Robustheit}

Im vorangegangenen Abschnitt wurden die Freiheitsgrade herausgearbeitet, welche sich beim Entwurf von FIOs für das nominale System im nicht-quadratischen Fall ergeben. Ziel ist es im Weiteren, diese zu nutzen, um die Robustheit der Fehlerisolation für das unsichere System der Form (2) zu optimieren, d.h. das Entwurfsziel (8) für alle Systeme aus $\mathcal{N}$ möglichst gut zu erfüllen.

\subsection{Optimierung der Isolationseigenschaft}

Zur Optimierung der Isolationseigenschaft wird zunächst die Partialbruchzerlegung von (7a) betrachtet, welche sich unter Annahme $6 \mathrm{zu}$

$$
\boldsymbol{G}_{\boldsymbol{r} f, l}(s)=\sum_{k=1}^{2 n} \frac{\boldsymbol{V} \tilde{\boldsymbol{C}}_{l} \tilde{\boldsymbol{v}}_{B_{k, l}} \cdot \tilde{\boldsymbol{w}}_{B_{k, l}}^{\top}\left(\tilde{\boldsymbol{E}}_{a, l}-\tilde{\boldsymbol{L}} \tilde{\boldsymbol{E}}_{s, l}\right)}{\boldsymbol{s}-\lambda_{B_{k}}}+\boldsymbol{V} \tilde{\boldsymbol{E}}_{s, l}
$$

ergibt. Beachtenswert ist, dass sich im Gegensatz zum nominalen Fall (12) bzw. (13) kein Ersatzsystem der Ordnung $n$ ergibt, sondern ein Gesamtsystem der Ordnung $2 n$. Es ist ersichtlich, dass zwar der Entwurf eines FIOs für die nominale Systemdynamik auf Basis von (12) bzw. (13) als duales Problem zum Entkopplungsreglerentwurf aufgefasst werden kann. Bei der Betrachtung unsicherheitsbehafteter Systeme wird nun deutlich, dass diese Dualität lediglich mathematischer Natur ist, da es im Bereich des Reg- lerentwurfs keine Entsprechung zu (18) bzw. dem Gesamtsystem der Ordnung $2 n$ gibt.

Für die Eckpunkte des Polytops $\mathcal{M}$ werden nun die Linkseigenvektoren analysiert. Zerlegt man die Linkseigenvektoren des Gesamtsystems in $\tilde{\boldsymbol{w}}_{B_{i, j,}}^{\top}=\left[\boldsymbol{w}_{B_{i j, l, s}}^{\top} \boldsymbol{w}_{B_{i j, l, b}}^{\top}\right]$, so ergibt sich aus ihrer Definitionsgleichung

$$
\begin{aligned}
& {\left[\begin{array}{ll}
\boldsymbol{w}_{B_{i j, l, s}}^{\top} & \boldsymbol{w}_{B_{i j, l, b}}^{\top}
\end{array}\right]\left[\begin{array}{cc}
\lambda_{B_{i j}} \boldsymbol{I}_{n}-\boldsymbol{A}_{l} & \mathbf{0} \\
\mathbf{0} & \lambda_{B_{i j}} \boldsymbol{I}_{n}-\boldsymbol{A}_{0}
\end{array}\right]} \\
& =\boldsymbol{w}_{B_{i j, b,}^{\top}}^{\top} \boldsymbol{L}\left[\begin{array}{ll}
\boldsymbol{C}_{l} & -\boldsymbol{C}_{0}
\end{array}\right] .
\end{aligned}
$$

Aus dem zweiten Spaltenblock dieser Beziehung lässt sich

$$
\boldsymbol{w}_{B_{i j, l, b}}^{\top}\left(\lambda_{B_{i j}} \boldsymbol{I}_{n}-\boldsymbol{A}_{0}\right)=-\boldsymbol{w}_{B_{i j, l, b}}^{\top} \boldsymbol{L} \boldsymbol{C}_{0}
$$

ablesen. Da dies (15) entspricht, folgt $\boldsymbol{w}_{B_{i j, l, b}}^{\top}=\boldsymbol{w}_{B_{i, j},}^{\top}$. Aus dem ersten Spaltenblock von (19) ergibt sich entsprechend

$$
\boldsymbol{w}_{B_{i j, l, s}}^{\top}\left(\lambda_{B_{i j}} \boldsymbol{I}_{n}-\boldsymbol{A}_{l}\right)=\boldsymbol{w}_{B_{i j, l, b}}^{\top} \boldsymbol{L} \boldsymbol{C}_{l}=-\boldsymbol{p}_{i j}^{\top} \boldsymbol{C}_{l} .
$$

\subsubsection{Steuerbare Beobachtereigenwerte}

Damit ein Eigenwert nur in der $i$-ten Spalte von $\boldsymbol{G}_{r f, l}(s)$ als Pol auftaucht, ist aufgrund des dyadischen Produktes in (18) die Gleichung $\tilde{\boldsymbol{w}}_{B_{k, l}^{\top}}^{\top}\left(\tilde{\boldsymbol{E}}_{a, l}-\tilde{\boldsymbol{L}} \tilde{\boldsymbol{E}}_{s, l}\right)= \pm \boldsymbol{\phi}_{i}^{\top}$ zu erfüllen. Es lässt sich durch Einsetzen leicht zeigen, dass dies durch (16b) für den nominalen Fall erreicht wird. Für die Eckpunkte von $\mathcal{M}$ ist nun die größte betragsmäßige Abweichung zu minimieren, es ist also

$$
\underset{z_{i j}^{\top}}{\operatorname{minimiere}} \max _{l=1, \ldots, N}\left\|\tilde{\boldsymbol{w}}_{B_{i j, l}^{\top}}^{\top}\left(\tilde{\boldsymbol{E}}_{a, l}-\tilde{\boldsymbol{L}} \tilde{\boldsymbol{E}}_{s, l}\right) \mp \boldsymbol{\phi}_{i}^{\top}\right\|_{2}
$$

zu lösen. Dabei ist das Vorzeichen von $\phi_{i}^{\top}$ nicht entscheidend, da lediglich die Richtung von $\phi_{i}^{\top}$ relevant für die Zuweisung zu einer Spalte von $\boldsymbol{G}_{r f}(s)$ ist und nicht die Orientierung. Das Problem (22) wird also einmal für $+\boldsymbol{\phi}_{i}^{\top}$ und einmal für $-\boldsymbol{\phi}_{i}^{\top}$ gelöst. Für den Beobachterentwurf wird dasjenige $\boldsymbol{z}_{i j}^{\top}$ verwendet, welches zu einer geringeren Norm in (22) führt.

Mit der gleichen Zerlegung des Linkseigenvektors wie in (19) ergibt sich für $\tilde{\boldsymbol{w}}_{B_{k, l}}^{\top}\left(\tilde{\boldsymbol{E}}_{a, l}-\tilde{\boldsymbol{L}} \tilde{\boldsymbol{E}}_{s, l}\right)$ der äquivalente Ausdruck

$$
\begin{aligned}
\tilde{\boldsymbol{w}}_{B_{i j, l}}^{\top}\left(\tilde{\boldsymbol{E}}_{a, l}-\tilde{\boldsymbol{L}} \tilde{\boldsymbol{E}}_{s, l}\right) & =\boldsymbol{w}_{B_{i j, l, s}}^{\top} \boldsymbol{E}_{a, l}+\boldsymbol{w}_{B_{i j, l, b}}^{\top} \boldsymbol{L} \boldsymbol{E}_{s, l} \\
& =\boldsymbol{w}_{B_{i j, l, s}}^{\top} \boldsymbol{E}_{a, l}-\boldsymbol{p}_{i j}^{\top} \boldsymbol{E}_{s, l} .
\end{aligned}
$$

Hierin tauchen noch die beiden Größen $\boldsymbol{w}_{B_{i j, l, s}}^{\top}$ und $\boldsymbol{p}_{i j}^{\top}$ auf, die jedoch durch die Gleichungsbedingung (21) miteinan- 
der verknüpft sind. Weiterhin gilt wegen (16b) für den $\mathrm{Pa}-$ rametervektor

$$
\boldsymbol{p}_{i j}^{\top}=\left(\left[\begin{array}{ll}
\mathbf{0}^{\top} & \boldsymbol{\phi}_{i}^{\top}
\end{array}\right] \boldsymbol{\Pi}_{i j}^{+}+\boldsymbol{z}_{i j}^{\top} \cdot{ }^{\perp} \boldsymbol{\Pi}_{i j}\right)\left[\begin{array}{c}
\mathbf{0} \\
\boldsymbol{I}_{n_{y}}
\end{array}\right] .
$$

Mit Hilfe des Schurkomplement-Lemmas [1] lässt sich (22) formulieren als

$$
\begin{aligned}
& \underset{z_{i j}^{\top}, \boldsymbol{w}_{B_{i j, l, s}^{\top}}^{\top}, \gamma_{f}}{\operatorname{minimiere}} \gamma_{f} \text {, u.d.B.d. } \forall l=1, \ldots, N \\
& \text { (21), (24), } \\
& {\left[\begin{array}{cc}
\gamma_{f} & \boldsymbol{w}_{B_{i j, l, s}^{\top}}^{\top} \boldsymbol{E}_{a, l}-\boldsymbol{p}_{i j}^{\top} \boldsymbol{E}_{s, l} \mp \boldsymbol{\phi}_{i}^{\top} \\
\star & \boldsymbol{I}_{n_{f}}
\end{array}\right]>\mathbf{0 .}}
\end{aligned}
$$

Anzumerken ist, dass durch (25) lediglich die Eckpunkte von $\mathcal{M}$ abgedeckt werden. Für den Spezialfall, dass lediglich die Fehlereingangsmatrizen $\boldsymbol{E}_{a, l}$ und $\boldsymbol{E}_{s, l}$ mit Unsicherheiten behaftet sind, lässt sich diese Aussage verallgemeinern: Der Vektor $\boldsymbol{w}_{B_{i j, l, s}}^{\top}$ ist in diesem Fall konstant und da (25) mit einem gemeinsamen Vektor $\boldsymbol{z}_{i j}^{\top}$ und einer gemeinsamen Schranke $\gamma_{f}$ berechnet wird, lässt sich zeigen, dass die Schranke $\gamma_{f}$ für die gesamte konvexe Hülle von $\mathcal{M}$ gilt. Dies ist dadurch zu begründen, dass für den genannten Spezialfall in (25) lediglich lineare bzw. affine Abhängigkeiten von den Variablen auftauchen.

\subsubsection{Nicht-steuerbare Beobachtereigenwerte}

Im nominalen System gilt $\tilde{\boldsymbol{w}}_{B_{k, l}}^{\top}\left(\tilde{\boldsymbol{E}}_{a, l}-\tilde{\boldsymbol{L}} \tilde{\boldsymbol{E}}_{s, l}\right)=\mathbf{0}^{\top}$, was durch (16c) bzw. (16d) erreicht wird und sicherstellt, dass der zugehörige Eigenwert für das Paar $\left(\boldsymbol{A}_{0}-\boldsymbol{L} \boldsymbol{C}_{0}, \boldsymbol{E}_{a, 0}-\right.$ $\left.\boldsymbol{L E}_{s, 0}\right)$ nicht-steuerbar ist. Um diese Eigenschaft für die Eckpunkte von $\mathcal{M}$ möglichst gut zu erreichen, lässt sich für die nicht-steuerbaren Beobachtereigenwerte, die nicht zur Kompensation invarianter Nullstellen herangezogen werden, analog zu (22) das Optimierungsproblem

$$
\underset{\boldsymbol{z}_{k}^{\top}, \lambda_{B_{k}}}{\operatorname{minimiere}} \max _{l=1, \ldots, N}\left\|\tilde{\boldsymbol{w}}_{B_{k, l}}^{\top}\left(\tilde{\boldsymbol{E}}_{a, l}-\tilde{\boldsymbol{L}} \tilde{\boldsymbol{E}}_{s, l}\right)\right\|_{2}
$$

formulieren. Entsprechend (21) und (23) ist

$$
\begin{aligned}
\boldsymbol{w}_{B_{k, l, s}}^{\top}\left(\lambda_{B_{k}} \boldsymbol{I}_{n}-\boldsymbol{A}_{l}\right) & =-\boldsymbol{p}_{k}^{\top} \boldsymbol{C}_{l}, \\
\tilde{\boldsymbol{w}}_{B_{k, l}}^{\top}\left(\tilde{\boldsymbol{E}}_{a, l}-\tilde{\boldsymbol{L}} \tilde{\boldsymbol{E}}_{s, l}\right) & =\boldsymbol{w}_{B_{k, l, s}}^{\top} \boldsymbol{E}_{a, l}-\boldsymbol{p}_{k}^{\top} \boldsymbol{E}_{s, l}
\end{aligned}
$$

und für den Parametervektor $\boldsymbol{p}_{k}^{\top}$ gilt nach (16c) die Beziehung

$$
\boldsymbol{p}_{k}^{\top}=\boldsymbol{z}_{k}^{\top} \cdot{ }^{\perp}\left[\begin{array}{cc}
\lambda_{B_{k}} \boldsymbol{I}_{n}-\boldsymbol{A}_{0} & \boldsymbol{E}_{a, 0} \\
-\boldsymbol{C}_{0} & \boldsymbol{E}_{s, 0}
\end{array}\right] \cdot\left[\begin{array}{c}
\mathbf{0} \\
\boldsymbol{I}_{n_{y}}
\end{array}\right] .
$$

Damit lässt sich schließlich das Optimierungproblem (26) umschreiben $\mathrm{zu}$

$$
\begin{aligned}
& \underset{\boldsymbol{z}_{k}^{\top}, \boldsymbol{w}_{B_{k, l, s}^{\top}}^{\top}, \lambda_{B_{k}}, \gamma_{f}}{\operatorname{minimiere}} \gamma_{f}, \text { u.d.B.d. } \forall l=1, \ldots, N \\
& (27 \mathrm{a}),(28), \\
& {\left[\begin{array}{cc}
\gamma_{f} & \boldsymbol{w}_{B_{k, l, s}}^{\top} \\
\star & \boldsymbol{E}_{a, l}-\boldsymbol{p}_{k}^{\top} \boldsymbol{E}_{s, l} \\
\star & \boldsymbol{I}_{n_{f}}
\end{array}\right]>\mathbf{0},} \\
& \left\|\boldsymbol{z}_{k}^{\top}\right\|_{2}=1, \\
& \lambda_{B_{k}} \in \mathcal{D} \subset \mathbb{C}^{-} .
\end{aligned}
$$

Beachtenswert sind dabei die beiden letzten Nebenbedingungen. Da die Linkseigenvektoren und Parametervektoren skalierungsinvariant sind [26], wird durch (29d) die Bedingung $\boldsymbol{z}_{k}^{\top} \neq \mathbf{0}^{\top}$ ausgedrückt. Mittels (29e) wird der nichtsteuerbare Eigenwert $\lambda_{B_{k}}$ auf ein Gebiet $\mathcal{D}$ in der linken s-Halbebene beschränkt. Es lässt sich beispielsweise eine obere Schranke für den Realteil von $\lambda_{B_{k}}$ vorgeben, um zu langsames Abklingen von Anfangsauslenkungen zu verhindern. Ebenso ist eine Beschränkung des Betrages von $\lambda_{B_{k}}$ sinnvoll, um differenzierendem Verhalten vorzubeugen. Muss $\lambda_{B_{k}}$ zur Kompensation einer invarianten Nullstelle herangezogen werden, so entfällt $\lambda_{B_{k}}$ als Freiheitsgrad und (29e) muss nicht berücksichtigt werden. Im Gegenzug vergrößert sich wie aus Satz 1 ersichtlich die Dimension von $\boldsymbol{z}_{k}^{\top}$.

Bei (26) handelt es sich im Gegensatz zu (22) nicht um ein LMI-Problem, sondern um ein nicht-konvexes Optimierungsproblem. Aufgrund der geringen Anzahl an $n_{y}-n_{f}$ Freiheitsgraden lässt es sich dennoch numerisch effizient lösen [7], wobei die Konvergenz auf ein globales Optimum nicht garantiert ist.

\subsubsection{Stationäre Isolation}

Im stationären Fall gilt für das nominale System bei einer Beobachterparametrierung nach Satz 1 die Beziehung

$$
\begin{array}{r}
\boldsymbol{G}_{r f, 0}(s=0)=-V \tilde{\boldsymbol{C}}_{0}\left(\tilde{\boldsymbol{A}}_{0}-\tilde{\boldsymbol{L}} \tilde{\boldsymbol{C}}_{0}\right)^{-1} \cdot \ldots \\
\cdot\left(\tilde{\boldsymbol{E}}_{a, 0}-\tilde{\boldsymbol{L}} \tilde{\boldsymbol{E}}_{s, 0}\right)+\boldsymbol{V} \tilde{\boldsymbol{E}}_{s, 0}=S
\end{array}
$$

Um für die Eckpunkte von $\mathcal{M}$ die Abweichung zur vorgegebenen Diagonalmatrix $\boldsymbol{S}$ zu minimieren, wird

$$
\underset{Z_{V}}{\operatorname{minimiere}} \max _{l=1, \ldots, N}\left\|G_{r f, l}(s=0)-S\right\|_{2}
$$

angestrebt. Im Weiteren wird die Matrix

$$
\boldsymbol{\Gamma}=-\boldsymbol{V} \tilde{\boldsymbol{C}}_{l}\left(\tilde{\boldsymbol{A}}_{l}-\tilde{\boldsymbol{L}} \tilde{\boldsymbol{C}}_{l}\right)^{-1}
$$


eingeführt. Hierin lässt sich die Lösung für den nominalen Fall (16e) einsetzen, um eine explizite Abhängigkeit von dem verbleibenden Freiheitsgrad $\boldsymbol{Z}_{V}$ zu erreichen. Mittels des Schurkomplement-Lemmas [1] ergibt sich somit das LMI-Problem

$$
\begin{aligned}
& \underset{Z_{V}, \boldsymbol{\Gamma}, \gamma_{f}}{\operatorname{minimier}} \gamma_{f} \text { u.d.B.d. } \forall l=1, \ldots, N \\
& (16 \mathrm{e}), \\
& \boldsymbol{\Gamma}\left(\tilde{\boldsymbol{A}}_{l}-\tilde{\boldsymbol{L}} \tilde{\boldsymbol{C}}_{l}\right)=-\boldsymbol{V} \tilde{\boldsymbol{C}}_{l}, \\
& {\left[\begin{array}{cc}
\gamma_{f} \boldsymbol{I}_{n_{f}} & \boldsymbol{\Gamma}\left(\tilde{\boldsymbol{E}}_{a, l}-\tilde{\boldsymbol{L}} \tilde{\boldsymbol{E}}_{s, l}\right)+\boldsymbol{V} \tilde{\boldsymbol{E}}_{s, l}-\boldsymbol{S} \\
\star & \boldsymbol{I}_{n_{f}}
\end{array}\right]>\mathbf{0 .}}
\end{aligned}
$$

Im Spezialfall, dass lediglich $\boldsymbol{E}_{a, l}$ und $\boldsymbol{E}_{s, l}$ mit Unsicherheiten behaftet sind, gilt die ermittelte Schranke $\gamma_{f}$ wegen der linearen bzw. affinen Abhängigkeit von den Variablen $\boldsymbol{\Gamma}$ und $Z_{V}$ nicht nur für die Eckpunkte von $\mathcal{M}$, sondern für die gesamte konvexe Hülle, obwohl nur endliche viele Nebenbedingungen berücksichtigt werden.

\subsection{Minimierung des Einflusses der Stellgrößen}

In Abschnitt 4.1 wurden die vorhandenen Entwurfsfreiheitsgrade dazu genutzt, die Isolationseigenschaft des FIOs möglichst robust bzgl. unsicherer Systemparameter zu gestalten. Im Fokus stand also (8a). In diesem Abschnitt erfolgt nun die Betrachtung von (8b), d.h. die Minimierung des Einflusses der Stellgrößen $\boldsymbol{u}$ auf die generierten Residuen.

\subsubsection{Beobachtereigenwerte}

Anhand von (12) wird deutlich, dass die Stellgrößen im nominalen Fall keinerlei Einfluss auf die Residuengenerierung haben und somit alle Beobachtereigenwerte bzgl. $\left(\boldsymbol{A}_{0}-\boldsymbol{L} \boldsymbol{C}_{0}, \boldsymbol{B}_{0}-\boldsymbol{L} \boldsymbol{D}_{0}\right)$ nicht-steuerbar sind. Führt man ana$\log \mathrm{zu}$ (18) eine Partialbruchzerlegung von $\boldsymbol{G}_{r u, l}(s)$ durch, so ist dementsprechend $\left\|\tilde{\boldsymbol{w}}_{B_{i, j},}^{\top}\left(\tilde{\boldsymbol{B}}_{l}-\tilde{\boldsymbol{L}} \tilde{\boldsymbol{D}}_{l}\right)\right\|_{2}$ zu minimieren. Benötigt wird an dieser Stelle also die Darstellung des zu minimierenden Terms in Abhängigkeit von den freien $\mathrm{Pa}$ rametern $\boldsymbol{z}_{i j}^{\top}$. Es ergibt sich

$$
\begin{aligned}
& \tilde{\boldsymbol{w}}_{B_{i j, l}}^{\top}\left(\tilde{\boldsymbol{B}}_{l}-\tilde{\boldsymbol{L}} \tilde{\boldsymbol{D}}_{l}\right) \\
& =\left[\begin{array}{ll}
\boldsymbol{w}_{B_{i j, l, s}}^{\top} & \boldsymbol{w}_{B_{i j, l, b}}^{\top}
\end{array}\right]\left[\begin{array}{c}
\boldsymbol{B}_{l} \\
\boldsymbol{B}_{0}-\boldsymbol{L}\left(\boldsymbol{D}_{0}-\boldsymbol{D}_{l}\right)
\end{array}\right] \\
& =\boldsymbol{w}_{B_{i j, l, s}}^{\top} \boldsymbol{B}_{l}+\boldsymbol{w}_{B_{i j, l, b}}^{\top} \boldsymbol{B}_{0}+\boldsymbol{p}_{i j}^{\top}\left(\boldsymbol{D}_{0}-\boldsymbol{D}_{l}\right) \\
& =\boldsymbol{w}_{B_{i j, l, s}}^{\top} \boldsymbol{B}_{l}+\left[\begin{array}{ll}
\boldsymbol{w}_{B_{i j, 0}}^{\top} & \boldsymbol{p}_{i j}^{\top}
\end{array}\right]\left[\begin{array}{c}
\boldsymbol{B}_{0} \\
\boldsymbol{D}_{0}-\boldsymbol{D}_{l}
\end{array}\right],
\end{aligned}
$$

wobei erneut $\boldsymbol{w}_{B_{i j, j, b}}^{\top}=\boldsymbol{w}_{B_{i j, 0}}^{\top}$ und $\boldsymbol{p}_{i j}^{\top}=-\boldsymbol{w}_{B_{i j, 0}}^{\top} \boldsymbol{L}$ ausgenutzt werden. Um eine explizite Abhängigkeit von $\boldsymbol{z}_{i j}^{\top}$ zu erhalten, wird (16b) eingesetzt, weiterhin gilt (21). Gelöst werden muss also analog zu (25) das Optimierungsproblem

$$
\begin{aligned}
& \underset{z_{i j}^{\top}, w_{B_{i j, l, s}}^{\top}, \gamma_{u}}{\operatorname{minimiere}} \gamma_{u} \text {, u.d.B.d. } \forall l=1, \ldots, N \\
& \text { (16b), (21), (24), }
\end{aligned}
$$

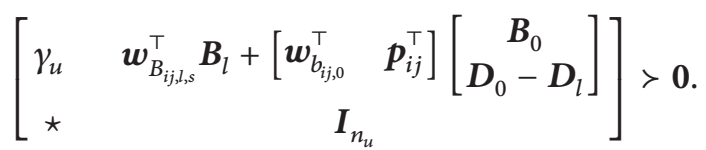

Für nicht-steuerbare Beobachtereigenwerte entspricht das Vorgehen Abschnitt 4.1.2.

\subsubsection{Stationärer Einfluss der Stellgrößen}

Analog zu Abschnitt 4.1.3 lässt sich der stationäre Einfluss der Stellgrößen auf die Residuen betrachten. Da gemäß (8) die Stellgrößen im Idealfall keine Wirkung auf die Residuen haben, ergibt sich

$$
\underset{Z_{V}}{\operatorname{minimiere}} \max _{l=1, \ldots, N}\left\|G_{r u, l}(s=0)\right\|_{2} .
$$

Entsprechend (33) lässt sich dies als LMI-Problem der Form

$$
\begin{aligned}
& \underset{Z_{V}, \Gamma, \gamma_{u}}{\operatorname{minimiere}} \gamma_{u} \text { u.d.B.d. } \forall l=1, \ldots, N \\
& (16 \mathrm{e}),(33 \mathrm{c}), \\
& {\left[\begin{array}{cc}
\gamma_{u} \boldsymbol{I}_{n_{f}} & \boldsymbol{\Gamma}\left(\tilde{\boldsymbol{B}}_{l}-\tilde{\boldsymbol{L}} \tilde{\boldsymbol{D}}_{l}\right)+\boldsymbol{V} \tilde{\boldsymbol{D}}_{l} \\
\star & \boldsymbol{I}_{n_{u}}
\end{array}\right]>\mathbf{0}}
\end{aligned}
$$

schreiben. Alternativ $\mathrm{zu}$ (37) lässt sich mittels des Bounded-Real-Lemmas [1] auch eine LMICharakterisierung der größten Verstärkung von $G_{r u}(s)$ im Sinne der $\mathcal{H}_{\infty}$-Norm angeben und optimieren.

\subsection{Zusammenfassung der Entwurfsmethodik}

In den Abschnitten 4.1 und 4.2 wurden die Optimierungsziele hinsichtlich der Isolationseigenschaft und der Unterdrückung des Einflusses von Stellgrößen formuliert. Dabei stellen beispielsweise (25) und (35) konkurrierende Ziele bzgl. der Wahl des Freiheitsgrades $\boldsymbol{z}_{i j}^{\top}$ dar. Um beiden Kriterien Rechnung zu tragen, wird für die Auslegung des FIOs eine gewichtete Summe der jeweiligen Schranken als 
Optimierungskriterium herangezogen. Für die steuerbaren Beobachtereigenwerte ist also das LMI-Problem

$$
\begin{aligned}
& \underset{z_{i j}^{\top}, \boldsymbol{w}_{B_{i j}, l, v^{\top}}, \gamma_{f}, \gamma_{u}}{\operatorname{minimiere}} \alpha_{f} \gamma_{f}+\alpha_{u} \gamma_{u} \text {, u.d.B.d. } \\
& \text { (16b), (21), (24), (25c), (35c) } \forall l=1, \ldots, N
\end{aligned}
$$

zu lösen. Dabei stellen $\alpha_{f} \geq 0$ und $\alpha_{u} \geq 0$ skalare Gewichtungsfaktoren dar, mit denen beim Entwurf zwischen der Trennschärfe der Isolation und der Unterdrückung des Einflusses von Störgrößen abgewogen werden kann.

Ebenso lässt sich bzgl. der nicht-steuerbaren Eigenwerte für (29) und das analoge Optimierungsproblem $\mathrm{zu}(35)$

$$
\begin{aligned}
& \underset{z_{k}^{\top}, w_{B_{k, l, s}}^{\top}, \lambda_{B_{k}}, \gamma_{f}, \gamma_{u}}{\operatorname{minimiere}} \alpha_{f} \gamma_{f}+\alpha_{u} \gamma_{u} \text {, u.d.B.d. } \\
& (16 \mathrm{c}),(16 \mathrm{~d}),(27 \mathrm{a}),(28),(29 \mathrm{c})-(29 \mathrm{e}),(35 \mathrm{c}) \\
& \forall l=1, \ldots, N
\end{aligned}
$$

schreiben. Dabei ist (35c) für die nicht-steuerbaren Eigenwerte $\lambda_{B_{k}}$ auszuwerten. Die nicht-steuerbaren Beobachtereigenwerte, welche invariante Nullstellen kompensieren, werden hier nicht gesondert betrachtet. Für sie ergibt sich strukturell das gleiche Optimierungsproblem wie (39), wobei der entsprechende Eigenwert nicht mitoptimiert wird.

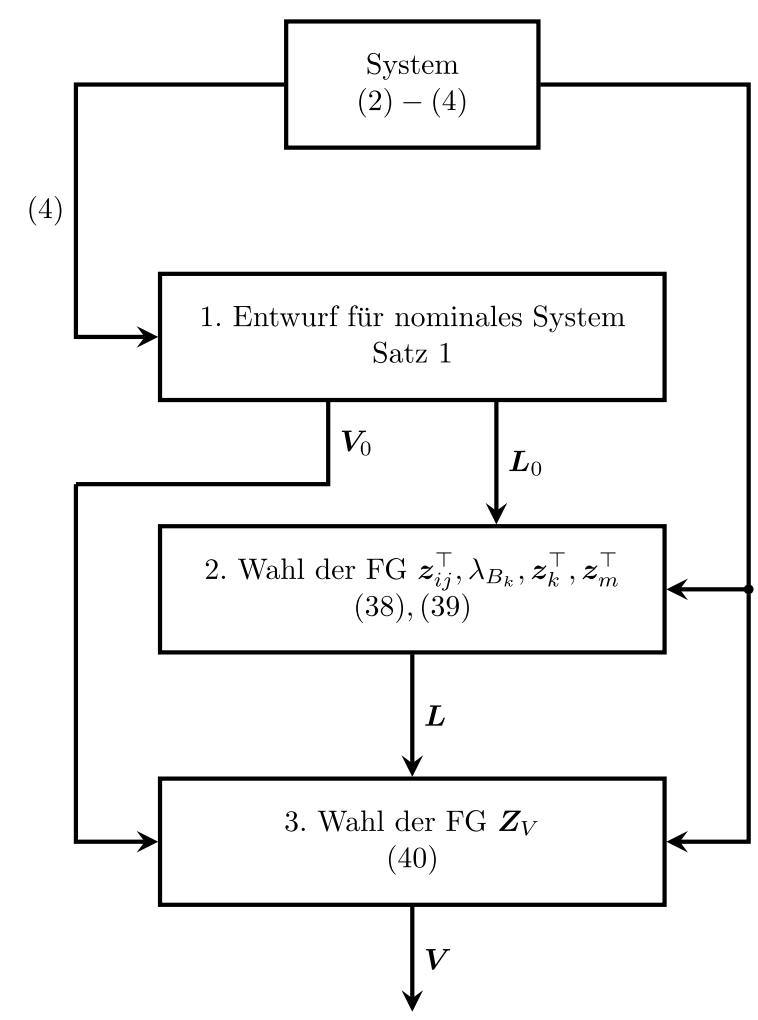

Abbildung 2: Schematische Darstellung des Entwurfsverfahrens.
Zur Wahl von $Z_{V}$ und damit zur Bestimmung des Nachfilters $\boldsymbol{V}$ lassen sich die LMI-Probleme (33) und (37) kombinieren $\mathrm{zu}$

$$
\begin{aligned}
& \underset{Z_{V}, \Gamma, \gamma_{f}, \gamma_{u}}{\operatorname{minimiere}} \alpha_{f} \gamma_{f}+\alpha_{u} \gamma_{u} \text {, u.d.B.d. } \\
& \text { (16e), (33c), (33d), (37c). }
\end{aligned}
$$

Abbildung 2 fasst das Entwurfsverfahren schematisch zusammen. Im ersten Schritt wird mittels Satz 1 der Entwurf für das nominale System durchgeführt, woraus sich $\boldsymbol{L}_{0}$ und $\boldsymbol{V}_{0}$ ergeben. Auf Basis der Unsicherheitsbeschreibung (2), (3) werden dann im zweiten Schritt durch Lösung der Optimierungsprobleme (38), (39) die Freiheitsgrade $\boldsymbol{z}_{i j}^{\top}, \boldsymbol{z}_{k}^{\top}$ und $\boldsymbol{z}_{m}^{\top}$ entworfen. Damit ist die gesuchte Beobachtermatrix $\boldsymbol{L}$ bereits parametiert. Im dritten Schritt wird auf dieser Grundlage das Optimierungproblem (40) gelöst, woraus sich schließlich die Nachfiltermatrix $\boldsymbol{V}$ ergibt.

Anmerkung 2. In der vorgestellten Form berücksichtigt das Entwurfsverfahren nur die Beobachtereigenwerte und die stationäre Isolation bzw. Stellgrößenunterdrückung. Es ist darüber hinaus möglich, auch den Einfluss der Streckeneigenwerte zu betrachten. Da auf diese über die Beobachtermatrix $\boldsymbol{L}$ kein Einfluss genommen werden kann, verbleibt die Anpassung von $\boldsymbol{V}$, um die Wirkung der Streckeneigenwerte auf die generierten Residuen zu unterdrücken. Dies lässt sich bewerkstelligen, indem die Norm der Zählermatrix in den Partialbruchzerlegungen von $\boldsymbol{G}_{\boldsymbol{r} f, l}(s)$ (18) und $\boldsymbol{G}_{\boldsymbol{r} u, l}(s)$ minimiert wird. Dazu wird das Optimierungskriterium in (40) um zusätzliche (gewichtete) Terme erweitert. Auf eine detaillierte Betrachtung wird an dieser Stelle verzichtet.

Anmerkung 3. Unterliegt das System zusätzlich zu den parametrischen Unsicherheiten noch exogenen Störungen, so lässt sich der vorgestellt Entwurf ohne Weiteres ebenfalls anwenden. Wie bei der Unterdrückung des Einflusses von Stellgrößen lässt sich ein Optimierungsproblem analog $\mathrm{zu}$ (35) formulieren, welches die Beobachtereigenwerte näherungsweise nicht-steuerbar bzgl. der Störungen macht. Um auch die Streckeneigenwerte und den stationären Einfluss der Störungen zu berücksichtigen, lässt sich für das Nachfilter $V$ ein Optimierungsproblem entsprechend (37) formulieren. Alternativ zur stationären Verstärkung lässt sich dabei mithilfe des Bounded-Real-Lemmas [1] auch eine Optimierung der größten Störverstärkung im Sinne der $\mathcal{H}_{\infty}$-Norm als LMI-Nebenbedingung formulieren. Die zu minimierende Schranke für die Störunterdrückung, $\gamma_{d}$ lässt sich dann mittels eines Gewichtungsfaktors $\alpha_{d}$ in die Gütekriterien der Optimierungsprobleme (38), (39) und (40) einbinden. 


\section{Beispiel}

Zur Verdeutlichung der Ergebnisse wird das Verfahren beispielhaft auf ein akademisches System dritter Ordnung angwendet. Das System verfügt über einen unsicheren $\mathrm{Pa}$ rameter $\varrho \in[-1,1]$, die beschreibenden Matrizen sind gegeben durch

$$
\begin{gathered}
A(\varrho)=\left[\begin{array}{ccc}
-1 & 2-0,5 \varrho & 4+0,2 \varrho \\
0 & -2 & 3+0,2 \varrho \\
0 & 0 & -3
\end{array}\right], \\
\boldsymbol{B}(\varrho)=\left[\begin{array}{cc}
0 & 0 \\
1-0,02 \varrho & 0,04 \varrho \\
0 & 4+0,06 \varrho
\end{array}\right], \\
\boldsymbol{E}_{a}(\varrho)=\left[\begin{array}{cc}
0 & 0 \\
1+0,2 \varrho & 0 \\
0 & 4-0,1 \varrho
\end{array}\right], \\
\boldsymbol{C}(\varrho)=\left[\begin{array}{ccc}
1+0,01 \varrho & 0 & -1 \\
0 & 1 & 0 \\
0 & -0,05 \varrho & 1
\end{array}\right],
\end{gathered}
$$

$\boldsymbol{D}(\varrho)=\mathbf{0}$ und $\boldsymbol{E}_{s}(\varrho)=\mathbf{0}$. Das nominale System (4) ergibt sich daraus mit $\varrho=0$ und die Eckpunkte des durch (2) definierten Polytops berechnen sich durch $\varrho=-1$ bzw. $\varrho=1$.

Für das System gilt $\delta_{1}=\delta_{2}=1$ und ferner sind die Annahmen 1-5 erfüllt. Es wird zunächst gemäß Satz 1 ein FIO für das nominale System entworfen. Für eine schnelle Fehlererkennung werden die Pole der Übertragungskanäle zu
$\lambda_{B_{1,1}}=-10$ und $\lambda_{B_{2,1}}=-12$ gewählt. Da das System keine invariante Nullstelle aufweist, kann der nicht-steuerbare Beobachtereigenwert frei gewählt werden, er wird für die initiale Beobachterparametrierung bei $\lambda_{B_{3}}=-8$ platziert. Als stationäre Verstärkung für die beiden Übertragungskanäle wird $z_{1}=z_{2}=1$ vorgegeben, die übrigen Freiheitsgrade werden zu $\boldsymbol{z}_{11}^{\top}=\boldsymbol{z}_{21}^{\top}=0, \boldsymbol{z}_{3}=1, \boldsymbol{Z}_{V}=\mathbf{0}$ gesetzt. $\mathrm{Zu}$ beachten ist dabei, dass wegen $n_{y}-n_{f}=1$ die Vektoren $\boldsymbol{z}_{i j}^{\top}$ bzw. $\boldsymbol{z}_{k}^{\top}$ zu Skalaren entarten. Es ergibt sich

$$
\boldsymbol{L}_{0}=\left[\begin{array}{ccc}
7,0 & 2,0 & 11,0 \\
-0,431 & 8,0 & 2,569 \\
-1,539 & 0 & 7,462
\end{array}\right], \boldsymbol{V}_{0}=\left[\begin{array}{ccc}
0 & 10,0 & 0 \\
-1,5 & 0 & 1,5
\end{array}\right]
$$

In Abbildung 3a ist die Sprungantwort von $\boldsymbol{G}_{r f}(s)$ für verschiedene Werte von $\varrho$ dargestellt. Während für $\varrho=0$ erwartungsgemäß eine ideale Fehlerisolation erreicht wird, zeigt sich für die Extremfälle $\varrho=-1$ bzw. $\varrho=1$ eine Abweichung in der stationären Verstärkung der Übertragungsfunktion $g_{1,1}(s)$ und eine deutliche Beeinflussung des ersten Residuums durch den zweiten Fehler. Darüber hinaus zeigt die Analyse der Sprungantwort von $G_{r u}(s)$ (s. Abbildung 4a), dass der Eingang $u_{2}$ für $\varrho \neq 0$ das Residuum $r_{1}$ stark beeinflusst. In diesem Fall ist also weder eine Fehlerdetektion noch eine -isolation möglich.

Für die Optimierung des FIOs sind lediglich die Parameter $\alpha_{f}$ und $\alpha_{u}$ sowie ein zulässiges Gebiet für den nichtsteuerbaren Beoabchtereigenwert zu wählen. Im Beispiel
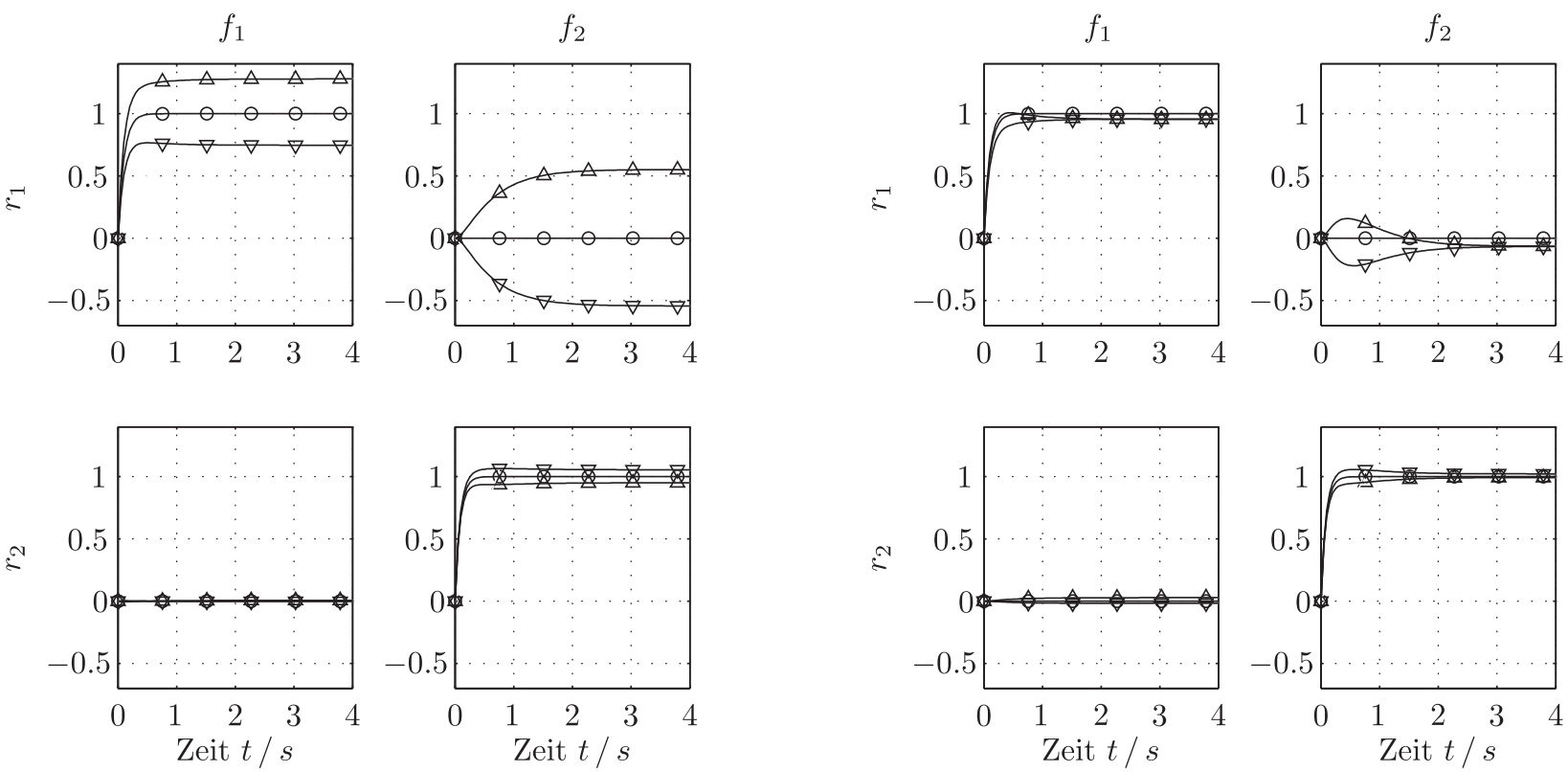

(a) Sprungantwort $\boldsymbol{G}_{\boldsymbol{r} f}(s)$ mit initialem Beobachter (०: nominales System, $\Delta: \varrho=1, \nabla: \varrho=-1$ )
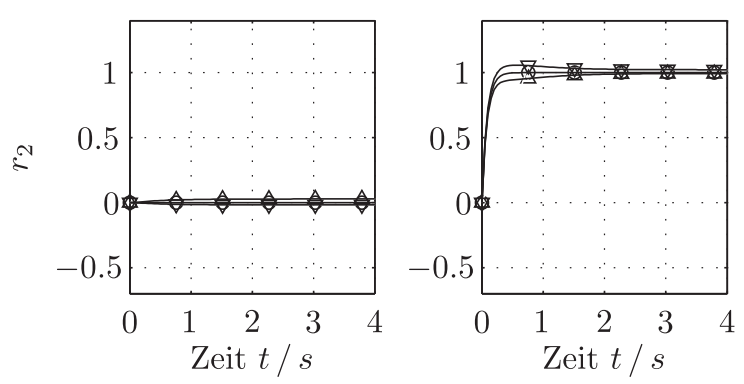

(b) Sprungantwort $\boldsymbol{G}_{r f}(s)$ mit optimiertem Beobachter (o: nominales System, $\triangle: \varrho=1, \nabla: \varrho=-1$ ) 

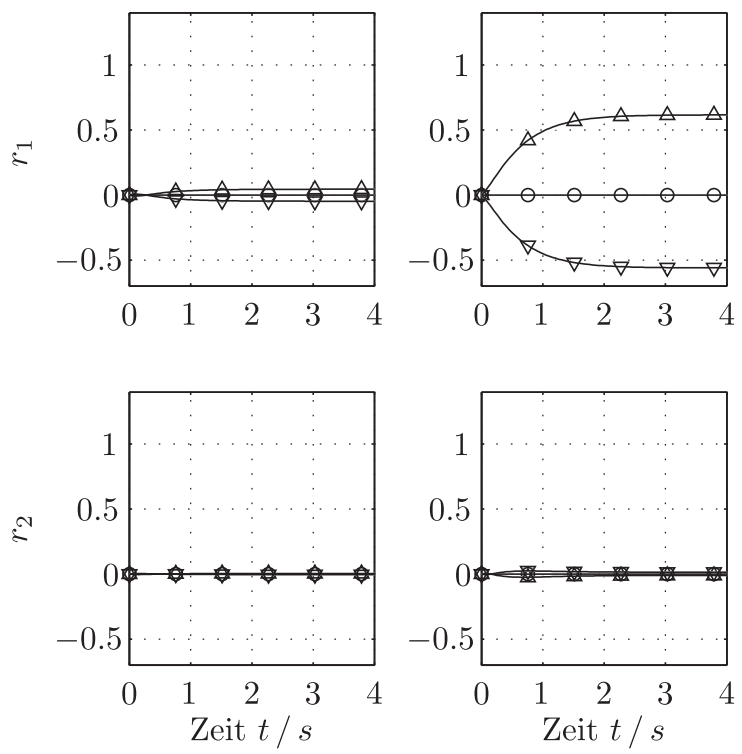

(a) Sprungantwort $\boldsymbol{G}_{\boldsymbol{r u}}(s)$ mit initialem Beobachter (०: nominales System, $\Delta: \varrho=1, \nabla: \varrho=-1$ )

Abbildung 4: Sprungantworten von $\boldsymbol{G}_{r u}(s)$.

werden $\alpha_{f}=2$ und $\alpha_{u}=1$ gewählt, die Diagonalstruktur von $\boldsymbol{G}_{r f}(s)$ wird also gegenüber der Unterdrückung des Einflusses von Stellgrößen auf die Residuen priorisiert. Als zulässiges Gebiet für den Eigenwert $\lambda_{B_{3}}$ wird $\mathcal{D}=\{\lambda \in$ $\mathbb{C} \mid-100<\mathfrak{R e}(\lambda)<-1\}$ gesetzt. Durch Lösen von (38) sowie (39) und Einsetzen in (16a) ergibt sich die gesuchte Beobachtermatrix $\boldsymbol{L}_{\mathrm{opt}}$. Anschließend wird durch Lösen von (40) die zugehörige optimierte Nachfiltermatrix $V_{\text {opt }}$ berechnet und es ist

$$
\begin{aligned}
\boldsymbol{L}_{\mathrm{opt}} & =\left[\begin{array}{ccc}
11,877 & 2,0 & 15,877 \\
1,437 & 8,0 & 4,437 \\
0,043 & 0 & 9,043
\end{array}\right], \\
\boldsymbol{V}_{\mathrm{opt}} & =\left[\begin{array}{ccc}
22,894 & 10,0 & 22,894 \\
-3,223 & 0 & -0,223
\end{array}\right] .
\end{aligned}
$$

Die auftretenden LMI-Probleme wurden dabei mithilfe des Interface YALMIP [20] und des Solvers SeDuMi [28] gelöst. Die Abbildung $3 \mathrm{~b}$ und $4 \mathrm{~b}$ zeigen die sich ergebenden Sprungantworten von $\boldsymbol{G}_{r f}(s)$ und $\boldsymbol{G}_{r u}(s)$. Aus Abbildung 3 ist ersichtlich, dass der optimierte Beobachter nicht nur wesentlich besser in der Lage ist, bei vorhandenen Unsicherheiten die gewünschte stationäre Verstärkung sicherzustellen. Es werden insbesondere auch die unerwünschten Kopplungen zwischen dem Fehler $f_{2}$ und dem Residuum $r_{1}$ reduziert. Wie aus Abbildung 4 hervorgeht, hat der Eingang $u_{1}$ für den optimierten Beobachter einen etwas stärkeren Einfluss auf das Residuum $r_{1}$, insgesamt wird der Einfluss der Stellgrößen auf die generierten Residuen jedoch deutlich reduziert.
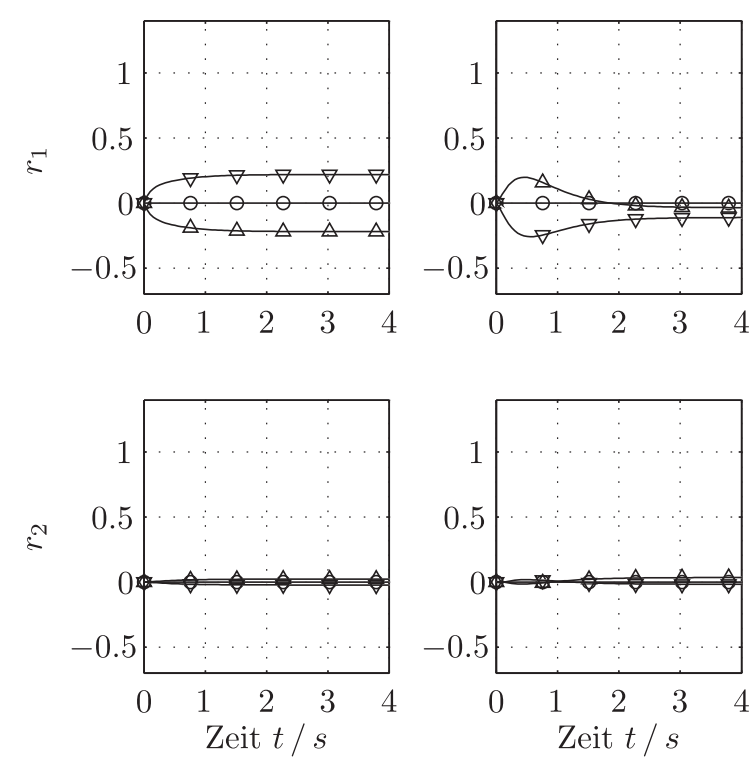

(b) Sprungantwort $\boldsymbol{G}_{\boldsymbol{r} \boldsymbol{u}}(s)$ mit optimiertem Beobachter (o: nominales System, $\triangle: \varrho=1, \nabla: \varrho=-1$ )

\section{Zusammenfassung}

Der Beitrag beschäftigt sich mit den zusätzlichen Entwurfsfreiheitsgraden für Fehlerisolationsbeobachter, welche sich gegenüber dem quadratischen Fall in Systemen mit mehr Sensoren als potentiellen Fehlern ergeben. Es wurde gezeigt, wie sich diese Freiheitsgrade systematisch nutzen lassen, um die Robustheit bzgl. unsicherer, beschränkter Systemparameter zu steigern. Hierfür ließen sich auf Basis der Vollständigen Modalen Synthese jeweils Optimierungsprobleme für die Auslegung der Freiheitsgrade ableiten, wobei für die nominale Systemdynamik stets eine exakte Fehlerisolation gewährleistet ist.

Eine Anwendung des vorstellten Verfahrens bietet sich in der Fehlerisolation von nichtlinearen Systemen, welche in der Nähe eines Arbeitspunktes betrieben werden. Linearisiert man die Systemdynamik um den Arbeitspunkt und entwirft einen linearen FIO mit der vorgestellten Methodik, so kann die gesteigerte Robustheit bzgl. parametrischer Unsicherheiten den Arbeitsbereich des FIOs vergrößern.

Danksagung: Diese Arbeit wurde unterstützt von der Deutsche Telekom Stiftung (www.telekom-stiftung.de).

Eingang 18. Januar 2013; angenommen 29. Juli 2013. 


\section{Literatur}

1. Boyd, S.; Ghaoui, L. E.; Feron, E.; Balakrishnan, V.: Linear Matrix Inequalities in System and Control Theory. SIAM, 1994.

2. Chen, B.; Nagarajaiah, S.: Linear-matrix-inequality-based robust fault detection and isolation using the eigenstructure assignment method. Journal of Guidance, Control, and Dynamics 30 (2007), Nr. 6, S. 1831-1835.

3. Chen, J.; Patton, R. J.: Robust Model-Based Fault Diagnosis for Dynamic Systems. Kluwer Academic Publishers, 1999.

4. Descusse, J.; Lafay, J. F.; Malabre, M.: Solution to morgan's problem. In: IEEE Transactions on Automatic Control 33 (1988), Nr. 8, S. 732-739.

5. Ding, S. X.: Model-based Fault Diagnosis Techniques. Springer, 2008.

6. Falb, P.; Wolovich, W.: Decoupling in the design and synthesis of multivariable control systems. In: IEEE Transactions on Automatic Control 12 (1967), Nr. 6, S. 651-659.

7. Forsgren, A.; Gill, P. E.; Wright, M. H.: Interior methods for nonlinear optimization. In: SIAM Review 44 (2002), Nr. 4, S. 525597.

8. Frank, P. M.: Fault diagnosis in dynamic systems using analytical and knowledge-based redundancy - a survey and some new results. In: Automatica 26 (1990), Nr. 3, S. 459-474.

9. Frisk, E.; Nielsen, L.: Robust residual generation for diagnosis including a reference model for residual behavior. In: Automatica 42, 3 (2006), S. 437-445.

10. Gilbert, E. G.: Controllability and observability in multivariable control systems. In: SIAM Journal on Control 2, (1963), Nr. 1, S. $128-151$.

11. Herrera H.; A. N.; Lafay, J. F.: New results about morgan's problem. In: IEEE Transactions on Automatic Control 38 (1993), Nr. 12, S. 1834-1838.

12. Isermann, R.: Fault-Diagnosis Systems. Springer, 2006.

13. Jaimoukha, I.; Li, Z.; Mazars, E.: Fault isolation filter with linear matrix inequality solution to optimal decoupling. In: In Proceedings of the American Control Conference Minneapolis, 2006, S. 2339-2344.

14. Koussiouris, T.: A frequency domain approach to the block decoupling problem. International Journal of Control 32 (1980), Nr. 3, S. 443-464.

15. Li, Z.; Jaimoukha, I. M.: Observer-based fault detection and isolation filter design for linear time-invariant systems. In: International Journal of Control 82 (2009), Nr. 1, S. 171-182.

16. Liu, B.; Si, J.: Fault isolation filter design for linear timeinvariant systems. In: IEEE Transactions on Automatic Control 42 (1997), Nr. 5, S. 704-707.

17. Liu, G. P.; Patton, R. J.: Parametric state feedback controller design of multivariable systems. In: International Journal of Control 61 (1995), Nr. 6, S. 1457-1464.
18. Lohmann, B.: Partial decoupling of non-minimum phase systems by constant state feedback. In: Kybernetika 27 (1991), S. 436-445.

19. Lohmann, B.: Vollständige und teilweise Führungsentkopplung dynamischer Systeme durch konstante Zustandsrückführung, Teil 1. In: at - Automatisierungstechnik 39 (1991), Nr. 9, S. 329334.

20. Löfberg, J.: YALMIP : a toolbox for modeling and optimization in MATLAB. In: Proceedings of the IEEE International Symposium on Computer Aided Control Systems Design. Taipei, 2004, S. 284-289.

21. Mazars, E.; Jaimoukha, I.; Li, Z.; Zolotas, A.: Fault detection and isolation filter design for systems subject to polytopic uncertainties. In: Proceedings of the Mediterrenean Conference on Control and Automation, 2007, S. 1-5.

22. Meyer, C. D.: Matrix Analysis and Applied Linear Algebra. SIAM, 2001.

23. Park, J.; Rizzoni, G.; Ribbens, W. B.: On the representation of sensor faults in fault detection filters. In: Automatica 30 (1994), Nr. 11, S. 1793-1795.

24. Patton, R. J.; Chen, J.: On eigenstructure assignment for robust fault diagnosis. In: International Journal of Robust and Nonlinear Control 10 (2000), Nr. 14, S. 1193-1208.

25. Roppenecker, G.: Modal approach to parametric state observer design. In: Electronics Letters 22 (1986), Nr. 3, S. 118-120.

26. Roppenecker, G.: On parametric state feedback design. In: International Journal of Control 43 (1986), Nr. 3, S. 793-804.

27. Roppenecker, G.; Lohmann, B.: Vollständige Modale Synthese von Entkopplungsregelungen. In: at - Automatisierungstechnik 36 (1988), Nr. 11, S. 434-441.

28. Sturm, J. F.: Using sedumi 1.02, a matlab toolbox for optimization over symmetric cones. In: Optimization Methods and Software 11 (1999), Nr. 1-4, S. 625-653.

29. Wahrburg, A.; Adamy, J.: Beobachterbasierte fehlerisolation für nicht-quadratische systeme mit unsicheren parametern. In: GMA-Fachausschuss 1.40 "Theoretische Verfahren der Regelungstechnik”. Salzburg, 2012.

30. Wahrburg, A.; Adamy, J.: Parametric design of robust fault isolation observers for linear non-square systems. In: Systems \& Control Letters 62 (2013), Nr. 5, S. 420-429.

31. Wei, M.; Shen, D.: Pole assignment in the regular row-by-row decoupling problem. In: Automatica 49 (2013), Nr. 1, S. 349359.

32. Wei, M.; Wang, Q.; Cheng, X.: Some new results for system decoupling and pole assignment problems. In: Automatica 46 (2010), Nr. 5, S. 937-944.

33. Zhong, M.; Ding, S. X.; Lam, J.; Wang, H.: An LMI approach to design robust fault detection filter for uncertain LTI systems. In: Automatica 39 (2003), Nr. 3, S. 543-550. 


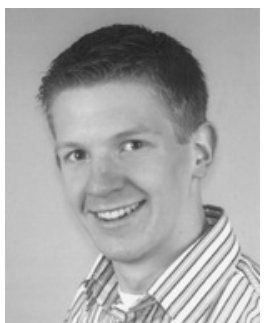

Dipl.-Ing. Arne Wahrburg

ABB AG, Forschungszentrum Deutschland, Wallstadter Str. 59, D-68526 Ladenburg, Tel.: +49-(0)6203-71-6166 arne.wahrburg@de.abb.com

Arne Wahrburg ist als Wissenschaftler am deutschen Forschungszentrum der ABB AG in der Gruppe Robotics \& Manufacturing tätig.

Verfügbar unter

lediglich die vom Gesetz vorgesehenen Nutzungsrechte gemäß UrhG

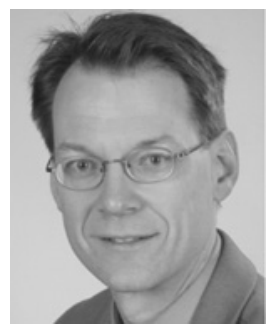

Prof. Dr.-Ing. Jürgen Adamy

Technische Universität Darmstadt, Institut für Automatisierungstechnik, Fachgebiet Regelungstheorie und Robotik, Landgraf-Georg-Str. 4, D-64283 Darmstadt, Tel.: +49-(0) 6151-16-3442

jadamy@rtr.tu-darmstadt.de

Jürgen Adamy ist Leiter des Fachgebiets Regelungstheorie und Robotik im Institut für Automatisierungstechnik der Technischen Universität Darmstadt. Hauptarbeitsgebiete: Regelungsverfahren, Computational Intelligence, autonome mobile Roboter. 